\title{
Sociología general en cárceles: sistematización de la experiencia educativa en contexto de encierro
}

\section{General sociology in prisons: systematization of an educational experience in prisons}

\author{
María Belén Roca Pamich \\ Facultad de Humanidades y Ciencias de la Educación- \\ Universidad Nacional de La Plata, Argentina \\ brocapamich@gmail.com
}

\section{RESUMEN:}

En este artículo vamos a recorrer algunas dimensiones centrales que presenta la educación universitaria en cárceles a partir de la experiencia en el dictado de la materia Sociología General en unidades penitenciarias provinciales durante los años 2017 y 2018. Es un intento por sistematizar estos años de trabajo generando una aproximación crítica sobre los desafíos pedagógicos y políticos que implica la educación en contextos de encierro, realizando un recorrido por las diferentes herramientas institucionales que se desarrollan en la Facultad de Humanidades y Ciencias de la Educación de la Universidad Nacional de La Plata.

Palabras Clave: Cárceles, Acceso a la Universidad, Estudiantes privados/as de su libertad, Sociología General.

\section{Abstract:}

In this article, we will go through some central dimensions of university education in prisons based on experience in dictating the general sociology subject in penitentiary units during the years 2017 and 2018 . This work is an attempt to systematize these years of work and generate a critical approach to the pedagogical and political challenges involved in education in confinement contexts, taking a tour of the different institutional tools that are developed in the Facultad de Humanidades y Ciencias de la Educación de la Universidad de La Plata.

KEYWORDS: Prisons, University access, Imprisoned Students, General Sociology, Education.

\section{CÁRCELES y UNIVERSIDAD}

La cárcel como institución de encierro en Argentina y especialmente en la provincia de Buenos Aires ha adquirido una centralidad enorme en las últimas décadas, tanto para el Estado como para los sectores populares: encontrándose cada vez más integrada a la vida de muchas personas que pasan por la cárcel, la visitan o trabajan dentro. El peso social que presenta como institución penal cobra relevancia año a año, tanto por la política de encarcelamiento masivo como por las consecuencias que genera.

La orientación de las políticas elaboradas desde diferentes esferas del Estado, entre las que resaltan el uso indiscriminado de la prisión preventiva, el policiamiento de la seguridad y la criminalización de la pobreza, han generado el colapso estructural del sistema carcelario argentino y bonaerense. Este colapso puede evidenciarse tanto en las condiciones de detención como en el crecimiento sostenido de personas privadas de su libertad. Según la estadística oficial la mayoría de las personas se encuentran detenidas en cárceles provinciales, mientras que la tasa de encarcelamiento a nivel nacional es de 186 en Buenos Aires es de 255 (CELS, 2018). Desde mediados de la década del 90 hasta la actualidad, la población encarcelada en Argentina se duplicó, pasando de 63 presos/as cada cien mil habitantes en 1996a 174 en el 2016 (Procuración Penitenciaria Nacional, 2017). En la provincia de Buenos Aires se da una dinámica similar pero con un crecimiento reciente muy importante: entre diciembre de 2012 y mayo de 2018 la tasa de personas detenidas pasó de 180 a 255 cada 100.000 habitantes en cárceles, alcaidías y comisarías (CELS, 2018). 
Esta política de encarcelamiento masivo tiene como sujeto de castigo predilecto a los jóvenes de sectores populares. La selectividad del sistema penal explica el hecho de que la gran mayoría de los y las detenidas pertenecen a las franjas más pobres de la población y estén cumpliendo condenas por delitos vinculados con la propiedad o el rédito económico (mayormente tráfico o venta de estupefacientes). En términos educativos y laborales solo el 34\% tiene completa la escuela primaria al momento de ingresar al sistema carcelario, el $41 \%$ se encontraba desocupado, y el 50\% no tenía ni oficio ni profesión (SNEEP, 2017). Estas características de las cárceles en Argentina y en la provincia de Buenos Aires han llevado a naturalizar la conformación clasista de las cárceles, que ha ido en paralelo a una justificación política del encierro como única opción (Viegas Barriga, 2012).

Miles de personas habitan y transitan las cárceles provinciales, instituciones libradas a la desidia del Estado, donde la violencia y las malas condiciones de vida son la norma. Llegar a la cárcel supone generalmente, para quienes la habitan, haber pasado antes por otras instituciones punitivas: comisarías, institutos de menores, alcaldías o la propia cárcel, en donde se van generando trayectorias vulnerables que vuelven aún más probable para algunos/as jóvenes terminar en prisión o reincidencia (Rodríguez Alzueta, 2012). Entrar en los circuitos de la justicia penal implica en Argentina verse degradado en la condición de ciudadano, ya que las desigualdades sociales se refuerzan, se fragmentan los lazos familiares, se empeora la calidad de vida, y por supuesto se interrumpen trayectorias educativas y laborales.

Como mencionamos anteriormente, la mayoría de los y las detenidas no han terminado la escuela primaria al momento de llegar a la cárcel y completan la escuela primaria y secundaria a lo largo de su condena. Esto genera trayectorias educativas sumamente fragmentadas y de baja calidad. Por lo que hemos podido observar durante estos años de trabajo en las cárceles, la desigualdad que implica cursar estudios primarios y/ o secundarios dentro de la cárcel se expresa tanto en contenidos como en habilidades vinculadas a la lectura, la escritura y la comprensión de textos. Esto se debe principalmente a que las escuelas dentro de las cárceles provinciales están en peores condiciones que las de afuera, cuentan con menos docentes que los necesarios, carecen de materiales para trabajar y no son jerarquizadas como un espacio importante en la vida de los y las presas por parte del Servicio Penitenciario Bonaerense. Se debe también a que cada vez que los y las detenidas son trasladadas de unidad penitenciaria por arbitrariedad del Servicio Penitenciario Bonaerense (SPB $)^{1}$, o por cambios de régimen, también cambian de escuela, lo cual en muchas ocasiones implica volver a cursar varias veces los mismos años, perder los papeles necesarios para pasar de nivel y cortar los procesos de aprendizaje, entre otras desventajas que traen los traslados.

En cuanto a la educación universitaria en las cárceles, resulta importante mencionar que así como sucede en la universidad en general, a pesar de los esfuerzos institucionales orientados para revertir esta situación, las posibilidades de iniciar estudios universitarios no están al alcance de todos y todas. Las y los estudiantes que están en condiciones de empezar estudios universitarios estando privados de su libertad representan un grupo reducido del total de la población, y esto se vuelve aún más complejo si tenemos en cuenta la escasa centralidad que se le da a la cuestión educativa dentro de las unidades. Quienes pueden vincularse con otros/as estudiantes universitarios mediante los centros de estudiantes $u$ otras actividades educativas necesitan encontrarse en ciertas posiciones particulares dentro de las lógicas de poder carcelaria además de tener el interés, estar en ciertos pabellones, no estar sancionados ni tener conflictos con estudiantes que formen parte del centro de estudiantes donde se desarrollan las actividades. Con esto nos referimos a que la educación universitaria en la cárcel expresa, al igual que fuera de ella, una posición de privilegio, que en el contexto carcelario adquiere dinámicas particularidades relacionadas a la posibilidad de participar de espacios educativos. Por otro lado, teniendo en cuenta que estudiar en la universidad requiere no sólo de mucha dedicación sino también de ciertas aptitudes y disposiciones, las trayectorias educativas previas, las condiciones de vulnerabilidad en las que viven y el aislamiento que implica la cárcel tampoco colaboran en generar las condiciones necesarias para empezar y mantener estudios universitarios. 
Sin embargo, a pesar de estas dificultades estructurales que implica la educación universitaria en contexto de encierro, desde diferentes facultades de la Universidad Nacional de La Plata ${ }^{2}$ y recientemente desde el rectorado de la Universidad, con la creación del Programa de Acompañamiento Universitario en Cárceles de la Prosecretaria de Derechos Humanos, se están desarrollando herramientas institucionales dedicadas a los y las estudiantes con el propósito de que tengan posibilidades de acceder a la universidad y que logren transitar las materias de cada una de las carreras.

En el caso de la Facultad de Humanidades y Ciencias de la Educación (FaHCE), desde el año 2008 se vienen realizando diferentes proyectos educativos en cárceles a través de proyectos de extensión y voluntariado a partir de los cuales se creó en el 2013 el Programa de Acompañamiento para Estudiantes Privados de su Libertad (PAEPL), dependiente de la Secretaría Académica. Este programa implicó una intervención constante y paulatina en unidades del SPB en donde se encuentran estudiantes privados de su libertad. A través de su acción ha logrado un crecimiento sostenido de los y las inscriptas en las carreras de Sociología e Historia y un acompañamiento a los/as estudiantes, logrando incrementar las posibilidades reales de estudiar, el avance en trámites burocráticos y en el rendimiento académico de los/as estudiantes, lo que se traduce en un aumento de las materias rendidas y en mayores niveles de aprendizaje.

El PAEPL tiene por objetivo incrementar la vinculación de la Facultad con los y las estudiantes privados/ as de su libertad inscriptos/as en las carreras de Sociología e Historia, como así también ampliar la cantidad de estudiantes. Podemos ver en el siguiente gráfico ${ }^{3}$ cómo han ido aumentando los/as alumnos inscriptos en dichas carreras desde el 2012 hasta el 2018.

\section{GRÁFICO 1}

Evolución inscripto/as a las carreras de Sociología e Historia (FaHCE) 2008-2018”

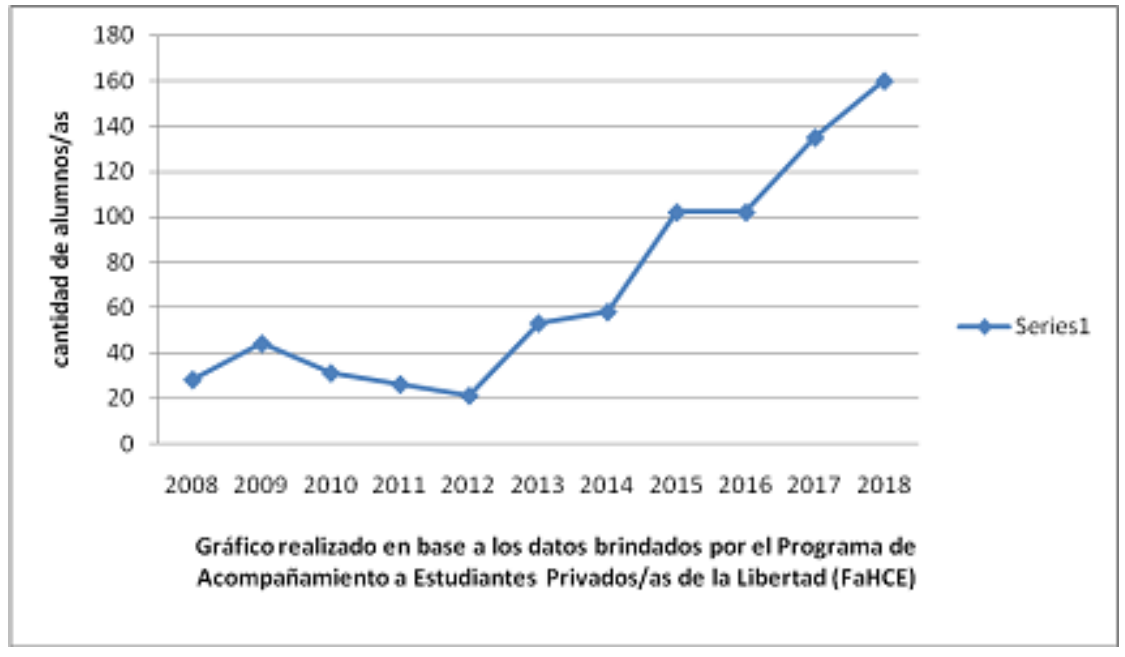

Si vemos en números absolutos, el total de inscriptos por año aumenta de 28 en el 2008 a 160 en el 2018. Este es un número muy significativo si tenemos en cuenta el tamaño de las carreras de Historia y Sociología en la Facultad de Humanidades y Ciencias de la Educación, donde por ejemplo a Sociología se inscriben cerca de doscientos estudiantes por año. Si bien muchos/as son solamente inscriptos y no han comenzado la carrera efectivamente, o no completaron la presentación de los papeles necesarios, el número total evidencia una política de ampliación del sector de estudiantes en cárceles. 


\section{GRÁFICO 2}

"Cantidad totales de inscriptos/s por carrera a Mayo del 2018"

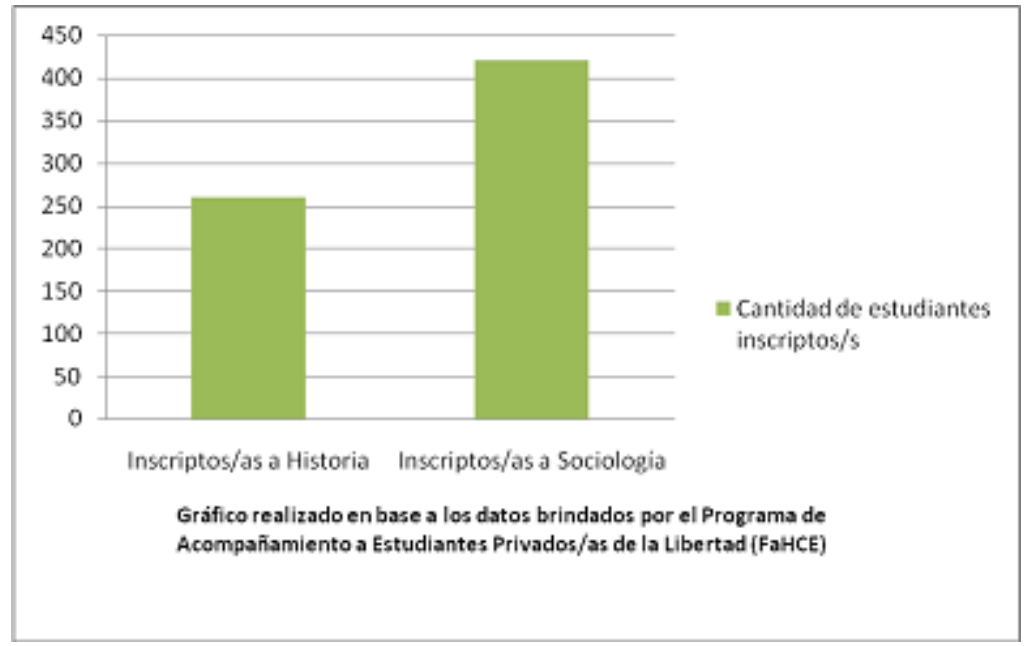

La política del PAEPL de ampliación de las inscripciones ha ido seguida también de un acompañamiento a los y las estudiantes que preparan materias, sumado a un aumento de la presencia docente en las unidades que ha generado que el número de alumnos/as que han concurrido a la FaHCE para rendir exámenes finales haya aumentado significativamente.

\section{GRÁFICO 3}

"Cantidad de exámenes finales rendidos en la FaHCE por cada estudiante a mayo 2018"

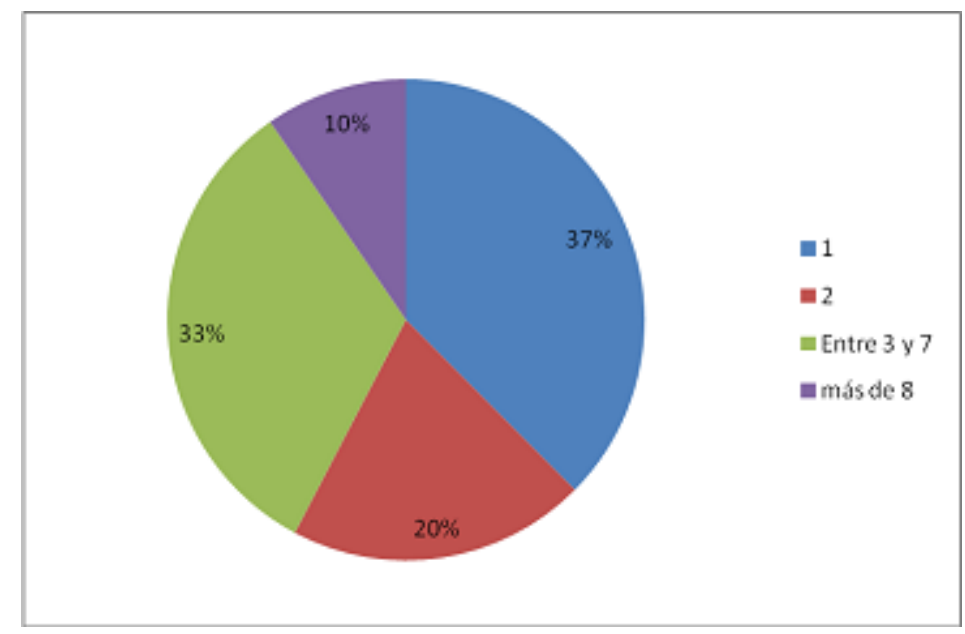

A Mayo del 2018, del total de alumnos/as de las carreras de Historia y Sociología 104 habían rendido un final y 34 estudiantes rindieron entre 3 y 7 finales (33\%). Podemos decir que, en general, los y las alumnas de las carreras de Sociología e Historia son ingresantes, y se encuentran transitando las primeras materias, habiendo rendido pocos finales. Durante el año 2017, de 135 inscriptos/as 20 rindieron exámenes finales durante ese año. También es interesante ver que hay un pequeño grupo que ha ido avanzando lentamente y ya se encuentra con más de ocho finales rendidos, es decir cerca del $30 \%$ de la carrera.

Teniendo en cuenta que la posibilidad de rendir un examen final en la FaHCE no depende únicamente de la voluntad de ir a rendir, es un número significativamente alto. Las dificultades para salir a rendir se deben a varias razones, una tiene que ver con los trámites administrativos necesarios para poder asistir a rendir un final libre a la FaHCE. Mientras que los requerimientos burocráticos necesarios para inscribirse a las carreras son muy flexibles a la hora de salir a rendir los finales los y las ingresantes requieren tener completo el trámite 
de inscripción: esto incluye presentar el analítico del secundario y el DNI. Sin embargo esto representa un problema burocrático importante para los y las detenidas, porque muchos/as no poseen analítico de finalización de la escuela secundaria aunque hayan terminado dentro de las cárceles, el documento nacional de identidad es sustraído por las autoridades del Servicio Penitenciario y es difícil conseguir copias, sumado al hecho de que algunos/as no tenían DNI al momento de ingresar a la cárcel. Estas dimensiones burocrático administrativas se vuelven sumamente importantes en el contexto de encierro, y en muchos casos atentan contra la iniciativa o la voluntad de los/as estudiantes de comenzar una carrera universitaria. Por ello, la labor del PAEPL es crucial en este tema para contrarrestar estas desigualdades estructurales de los y las estudiantes. Al mismo tiempo, otra dificultad que se agrega a la hora de salir a rendir los finales es la necesaria articulación burocrática administrativa entre la FaHCE, el Servicio Penitenciario Bonaerense y los juzgados penales para que las y los estudiantes logren salir a rendir a la Facultad. Una vez realizada la inscripción, la autorización necesaria para poder ir de la unidad penitenciaria a la FaHCE depende de los juzgados que siguen las causas penales de los y las estudiantes y de las posibilidades (humanas y materiales) con las que el SPB cuenta para realizar los traslados hasta la mesa de examen. Cuando estas autorizaciones y disponibilidades institucionales no están, el hecho de salir a rendir resulta excepcional.

\section{Sociología General en la CÁRCEL}

En este marco de intervención institucional en las cárceles, también los departamentos de Sociología y de Historia de la Facultad de Humanidades y Ciencias de la Educación comenzaron a involucrarse con las y los estudiantes privados/as de su libertad, a través de sus cátedras, en el dictado de clases teóricas dentro de las unidades, clases de consulta, la realización de talleres del curso de ingreso y charlas informativas sobre las carreras a estudiantes secundarios, entre otras actividades académicas.

Desde el año 2013 la cátedra de Sociología General viene dictando clases en las cárceles, a cargo de un conjunto rotativo de docentes; a partir del año 2017 se asignaron dos cargos docentes específicos que tienen como tarea enseñar la materia en ese ámbito. La modalidad implica el dictado de clases prácticas en un total de cuatro unidades penitenciarias durante un cuatrimestre, que hasta el momento fueron tres unidades de varones y una de mujeres, elegidas con el criterio de que concentren la mayor cantidad de inscriptos/as. Con esquemas similares a como ocurre con las comisiones de trabajos prácticos en la Facultad, se dan clases que incluyen actividades y trabajo con los textos, aunque no se valida como una cursada de trabajos prácticos sino que las y los estudiantes luego rinden examen final en modalidad libre. Actualmente, la cátedra de Sociología General es la única que tiene docentes dedicadas al dictado de clases durante todo un cuatrimestre en las unidades, aunque otras cátedras adoptan diferentes modalidades para realizar el acompañamiento a los y las alumnas que van preparando sus materias.

La materia de Sociología General tiene una relevancia muy importante para los y las estudiantes privadas de su libertad porque es el primer eslabón de un acompañamiento a los y las alumnas en su iniciación académica. Este acompañamiento académico que se realiza desde la cátedra de Sociología General, el Departamento de Sociología y el Programa de Acompañamiento a Estudiantes privados de su libertad (PAEPL), implica una inserción concreta en las cárceles por parte de la Facultad de Humanidades y Ciencias de la Educación para garantizar el derecho a la educación universitaria a un sector importante de la población cuyas posibilidades de acceder a ese derecho resultan coartadas.

Sociología General es una materia introductoria para alumnos/as de Historia y Sociología, y es una pieza fundamental en estos primeros pasos académicos, ya que al ser dictada semanalmente por docentes de la FaHCE logra una aproximación a lo que es estudiar una carrera universitaria en "la calle", noción que utilizan los/as detenidas para hablar del afuera carcelario. Así también por los contenidos que aborda, introduce a los y las alumnas en la sociología y en el pensamiento crítico en general, y cobra importancia como introducción teórica. Es por eso que entendemos que el recorrido de los y las estudiantes por la materia adquiere una 
relevancia inevitable, resultando una primera aproximación a nociones y saberes básicos necesarios para el desarrollo de sus estudios universitarios, brindando un panorama general sobre la sociología como ciencia social y una perspectiva para abordar distintos fenómenos sociales propios de las sociedades modernas. De esta forma se configura como una bisagra entre los saberes previos que traen los/as estudiantes y los contenidos que se buscan impartir para dar comienzo a la carrera. Este pasaje es un nodo crucial porque la distancia entre los saberes previos y los que brinda la materia es por demás importante para los y las estudiantes privadas de su libertad, una brecha que es necesario reconocer para no alejar a los y las estudiantes de los estudios dando por supuesto conocimientos que no están.

En cuanto al programa propuesto por la cátedra, la asignatura tiene como objetivo principal introducir a las y los alumnos en la perspectiva sociológica, brindar un primer acercamiento al surgimiento de la sociología como ciencia social, las bases teóricas elaboradas por sus clásicos para interpretar la sociedad moderna y a los diversos enfoques para aproximarse a problemáticas sociales de importancia. De ese modo, plantea adentrarse en los núcleos temáticos centrales de la sociología y vincularlos con problemáticas propias de la Argentina actual, utilizando textos de autores reconocidos (tanto clásicos como contemporáneos), comentaristas y también textos de divulgación sobre esos mismos temas.

Es por eso que en esta primera materia se busca que los alumnos adquieran algunos conocimientos básicos sobre la ruptura que hace la sociología sobre el sentido común, su especificidad como ciencia social y la manera particular de entender la relación entre el individuo y la sociedad. En este plano, la disciplina en general y la materia en particular, presenta un gran potencial para la desnaturalización de la realidad y el descubrimiento de los enlaces que anudan biografías individuales con condicionamientos históricos.

Considerando el hecho de que los y las alumnas privados de su libertad se encuentran en un contexto adverso para llevar adelante sus estudios, en procesos largos de acumulación de desventajas (Esping Andersen en Saraví, 2015), habiendo terminado sus estudios secundarios dentro de las instituciones penales, la fragmentación escolar es una dimensión a atender de forma prioritaria en el dictado de la materia. Una de las formas de contrarrestar esto es captando y conteniendo la heterogeneidad de recorridos de los/as estudiantes al momento de cursar la materia, y dándole lugar al desarrollo de habilidades escritas, orales y de comprensión lectora, que no se dan de forma natural sino que es necesario enseñarlas. Al mismo tiempo es importante a la hora de desarrollar la materia priorizar aspectos que hacen a la socialización académica. De esta manera se empalma con uno de los objetivos presentes en la política de ingreso del Departamento de Sociología, que se lleva adelante en el curso de ingreso de la FaHCE, que tiene que ver con apostar a la socialización académica para lograr mayores niveles de permanencia.

Las clases de sociología resultan una instancia elemental para que los alumnos/as incorporen y desarrollen las habilidades necesarias para poder avanzar y permanecer en su carrera universitaria. Por ello en las clases buscamos dedicarle una parte importante a los procesos de compresión de textos, brindando herramientas necesarias para su análisis y estudio. La enseñanza de técnicas de estudio se vuelve parte de nuestra labor docente, entendiendo que el desarrollo de las habilidades y disposiciones necesarias para el estudio son fundamentales para el dictado de una materia y no parte accesoria de los contenidos.

La posibilidad de estudiar una carrera universitaria y de asistir a clases estando detenidos/as resulta muy significativa para los y las estudiantes en tanto les brinda la posibilidad de generar cambios importantes en sus trayectorias educativas y de encierro, dando origen a un conjunto de expectativas de vinculación con la vida universitaria y el estudio, tanto durante el encierro como una vez en libertad. En este sentido, consideramos que es un gran desafío institucional que el acompañamiento y la educación en las cárceles sean satisfactorios para los y las estudiantes y no se convierta en otra experiencia educativa frustrada. Creemos que esto es posible a partir de una apuesta por generar las condiciones necesarias para que las expectativas depositadas en la educación superior sean posibles y logren cambios concretos en sus vidas cotidianas, con el reconocimiento del valor que tiene para ellos/as la educación y la institución universitaria. 


\section{Aspectos Del DiCTAdo de Clases en Ámbitos CARCELARios}

El contexto específico donde se desarrollan las clases, las particularidades de los alumnos y alumnas y los espacios físicos hacen que las clases de sociología en las cárceles tengan particularidades que son interesantes para poner de relieve.

Los espacios físicos donde se desarrollan las clases varían de unidad en unidad, y resultan de suma importancia para entender las particularidades del dictado de clases dentro de las unidades penitenciarias.

En algunas unidades penales existen mejores condiciones para dar una clase que en otras, siendo el espacio físico una variable fundamental para poder desarrollar una clase cómodamente, con cierto grado de silencio, sillas disponibles, buena luz, pizarrón, entre otras variables que hacen que una clase se pueda dictar o no. Dependiendo de cada unidad los espacios pueden ser más "educativos" o más "carcelarios", con esto nos referimos tanto a la espacialidad como a las lógicas que se desarrollan en las unidades. Estas pueden ser más propias del SPB, en donde la educación es entendida como un beneficio para algunos/as y no como un derecho a ser garantizado, o pueden estar más relacionadas a los espacios de centro de estudiantes o instituciones educativas en donde se busca generar las condiciones para que la educación tenga relevancia dentro de las cárceles.

En algunas unidades no hay espacios destinados a la educación universitaria y se utilizan las aulas de la primaria o la secundaria, por lo que la disputa por los lugares es moneda corriente, siendo siempre insuficientes. Sin embargo existen también espacios áulicos, en su mayoría pertenecientes a los centros de estudiantes que existen dentro de las cárceles y otorgan mejores condiciones para poder desarrollar las clases, donde hay cierta autonomía y gestión por parte de los y las estudiantes. Mencionamos estas características materiales porque son cruciales para el dictado de clases en las cárceles y sus variaciones repercuten en la instancia pedagógica.

Vinculada a la dimensión espacial entra en juego a la hora de dar clases el ambiente en el que se desarrolla. Como decíamos más arriba este puede ser más “educativo" o más "carcelario", teniendo en cuenta de igual forma todos se dan dentro de la cárcel y las reglas de la institución son determinantes para la realización de actividades en su interior. En algunas unidades las interrupciones por parte de las autoridades penitenciarias pueden volverse recurrentes durante el desarrollo de la clase, cortando las dinámicas educativas con lógicas propias de la cárcel como puede ser tomar lista, controlar los alumnos, retirarlos, entre otras prácticas institucionales. Por eso los espacios de los centros de estudiantes suelen ser más propicios para desarrollar las clases, aunque muchas veces depende más de la política de la unidad que los y las alumnas puedan asistir y la clase se pueda desarrollar sin interrupciones que de los espacios físicos.

Otra dimensión crucial a la hora de abordar las particularidades de la educación en las cárceles tiene que ver con las trayectorias biográficas de los y las alumnas, en su recorrido previo a la cárcel, sus experiencias educativas anteriores, y también las condiciones de vida dentro de la cárcel. Por un lado, sucede que las vivencias personales se cuelan con los contenidos que se ven en la materia, y de esa forma la cárcel toma lugar para ser analizada. En las cárceles de mujeres, las vivencias de las alumnas en el encierro son traídas con más frecuencia a las clases, por el aislamiento en el que se encuentran, por las características que adquiere la maternidad, en tanto algunas viven con sus hijos/as dentro de las unidades o porque sufren el hecho de no ver a sus hijos/as por largos periodos, como también por las violencias de género que padecen mientras se encuentran detenidas. ${ }^{4}$ Por otro lado, la cárcel irrumpe, como institución, en la dinámica de "lo educativo", cuando pasan lista, cuando son requisados/as para ir a sus pabellones, en el trato del penitenciario a los/ as estudiantes. Si durante la clase tal vez la dimensión carcelaria había quedado en un segundo plano, cobra realidad en pocos minutos. De esa manera "lo educativo" y "lo carcelario" se entrelazan y tensionan, y construyen espacios que en equilibrios inestables permiten el desarrollo educativo dentro de las cárceles.

Asimismo es necesario mencionar que el acceso a la educación en las cárceles es más limitado que en otros espacios sociales y se dificulta por el hecho de que es entendido por las autoridades judiciales y 
penitenciarias como un beneficio y no como un derecho. Esto quiere decir que quienes logran acceder a la educación universitaria dentro de la cárcel hacen un esfuerzo muy grande para sostenerlo en el tiempo y, como mencionamos anteriormente, son un pequeño porcentaje de la población encarcelada total. Esto tiene como consecuencia que los y las estudiantes valoren mucho las instancias de clases y respeten la labor de las profesoras y profesores; así, el reconocimiento a quienes vamos regularmente es constante y colabora en que las clases tengan buen clima de trabajo.

En relación a estas dimensiones podemos decir que las clases de Sociología General indefectiblemente se adaptan a las diferentes realidades que existen en las unidades penales donde se da la materia. Nos referimos tanto a la cantidad de alumnos y alumnas, número que varía entre las unidades penales, ${ }^{5}$ como al régimen de seguridad de cada penal, los espacios físicos disponibles, la voluntad de las autoridades para desarrollar las clases, las condiciones de vida de los y las detenidas, condiciones que son muy importantes a la hora de desarrollar la clase y que forman parte constitutiva del contexto específico.

Por último, es interesante mencionar que estas particularidades que hacen al dictado de clases en la cárcel vuelven imprescindible el diálogo con aquellos saberes prácticos y teóricos que los alumnos traen, revalorizando sus experiencias de vida y sus conocimientos específicos. Siendo que muchas de sus experiencias educativas anteriores han sido conflictivas, en tanto han terminado el colegio secundario de grandes o muchos han empezado el proceso de escolarización una vez en la cárcel, resulta fundamental no desconocer esos procesos e intentar revertirlos mediante la experiencia universitaria. Es por eso que forjar un interés en quienes se anotaron en las carreras de Historia y Sociología es de suma importancia, ya que permite tender un puente con el bagaje escolar previo, su experiencia de vida y su ingreso a la vida académica, la universidad y la materia Sociología General. Nuestra experiencia en contextos de encierro nos ha mostrado que este último punto resulta fundamental para que los y las presas adquieran mayor confianza en sí mismos a la hora de llevar adelante un proceso educativo y contrarrestar experiencias pasadas.

\section{APORTES PARA LA REFLEXIÓN POLÍTICO-PEDAGÓGICA}

Reflexionar sobre la educación universitaria en cárceles implica poner sobre la mesa la desigualdad que existe entre aquellos estudiantes que van a la Facultad en "la calle" - como le llaman los presos y las presas al afuera de prisión- y quienes asisten dentro de las unidades penitenciarias.

Como intentamos poner de manifiesto a lo largo del artículo, la educación en contextos de encierro pone de manifiesto las desigualdades de clase existentes en los sujetos que acceden a la universidad estando privados/ as de su libertad, a las cuales se le suman las desigualdades educativas. Dimensión central a la hora de pensar cómo lograr que las y los estudiantes accedan a los conocimientos que la carrera de Sociología y la materia de Sociología en general buscan impartir. Esta dificultad no es sólo propia de los ingresantes que se encuentran privados de su libertad, ya que también ha sido una problemática que comenzó a ser trabajada desde los diferentes claustros del departamento de sociología en pos de mejorar el ingreso y la permanencia de los/as estudiantes de sociología, como por ejemplo mediante la creación y la obligatoriedad del curso de ingreso. Herramienta que busca contrarrestar las desigualdades escolares que existen entre quienes se anotan en la Facultad.

Esta realidad se agudiza en las unidades penitenciarias en donde no sólo existe una desigualdad muy marcada en las trayectorias educativas sino también en las disposiciones culturales y sociales necesarias para atravesar un proceso educativo de alta complejidad como es una carrera universitaria. En este punto existe una relación tensa entre la dinámica de ampliación de derechos y la calidad educativa, siendo que muchas veces la excelencia académica va en detrimento de la inclusión mientras que la ampliación también puede significar empeorar la calidad educativa. En relación a la experiencia realizada entendemos que es necesario ampliar la oferta educativa para estudiantes privados de su libertad, apostando a garantizar el derecho a la educación, realizando un esfuerzo por brindar oportunidades de calidad mediante un acompañamiento 
paulatino que tenga en cuenta las necesidades particulares de los y las estudiantes privados/as de su libertad. Saber leer y escribir no resulta una obviedad para quienes están detenidos/as, aún entre quienes formalmente terminaron la escuela secundaria. Es por eso que el dictado de clases en contexto de encierro requiere no sólo la particularidad del contexto en donde se desarrolla, sus propias lógicas y dinámicas, sino también atender a los sujetos que son parte de esas clases. Brindar herramientas conceptuales para el análisis de textos académicos puede volverse un desafío por demás difícil si no se tiene en cuenta todo otro universo de saberes y habilidades que son necesarias para estudiar, comprender y analizar.

El reconocimiento político pedagógico de conocer a los y las estudiantes en sus particularidades se vuelve fundamental para romper con el formalismo educativo que desconoce a quienes tiene enfrente. Los y las estudiantes no son sólo mentes y cuerpos, las trayectorias de vida hacen a los y las estudiantes y a la clase, por lo cual deben estar en diálogo con la propuesta educativa. La política del reconocimiento es fundamental para que exista un vínculo pedagógico real y para que la experiencia educativa no colabore en aumentar las desigualdades preexistentes.

La experiencia de dictar Sociología General durante dos cuatrimestres nos permite decir que es muy importante para las clases el acompañamiento pedagógico dentro de las cárceles a los y las estudiantes de la Facultad. Es posible percibir el progreso entre los primeros encuentros y los últimos, además del desempeño de los/as estudiantes en los exámenes finales. En este sentido el Programa de Acompañamiento a Estudiantes Privados de su Libertad resulta clave para que las clases de sociología puedan ser clases de sociología y no un espacio donde se atiendan otras cuestiones acuciantes para los/as estudiantes. El acompañamiento semanal convierte a la institución en una presencia cotidiana, para la realización de trámites, el acceso a materiales, las consultas, y genera las condiciones para que las clases de sociología puedan desarrollar los contenidos propios de la materia.

Dar clases en las cárcel también implica obligadamente un ejercicio imaginativo, de creatividad pedagógica que impulse a los y las estudiantes, que permita que se interesen por la sociología, entender qué elementos tiene para aportar como ciencia social y poder valerse de los conocimientos que ofrece la disciplina para analizar tanto la realidad social en general como su situación personal. La clase es un momento que los/as estudiantes valoran mucho, sobre todo por la presencia de las profesoras, las explicaciones y actividades, ya que muchas veces trae aparejado olvidar por un rato las condiciones de vida con las que tienen que lidiar cotidianamente.

\section{Bibliografía}

Rodríguez Alzueta, E. (2012). Circuitos carcelarios: El encarcelamiento masivo-selectivo, preventivo y rotativo en la Argentina. Question, 1(36), 81-96.

Saraví, G. A. (2015). Juventudes fragmentadas: socialización, clase y cultura en la construcción de la desigualdad. México: FLACSO.

Viegas Barriga, F. (2012). Desencajados. Remanencias de pabellón en liberados de cárceles bonaerenses. En Barreneche O. y Oyhandy A. (comps.), Leyes, justicias e instituciones de seguridad en la provincia de Buenos Aires (siglos XIX a XXI), La Plata, Universidad Nacional de La Plata, Facultad de Humanidades y Ciencias de la Educación (pp. 321-351).

\section{INFORMES CONSULTADOS}

Dirección Nacional de Política Criminal, Informe Anual del Sistema Nacional de Estadísticas sobre Ejecución de la Pena, SNEEP. Año 2017.

Procuración Penitenciaria de la Nación. Informe Anual. Año 2017. 


\section{ArtíCulOS DE INTERNET}

CELS (2018). Cárcel y Sistema Penal. Disponible en: https://www.cels.org.ar/web/2018/07/\%e2\%80\%8breunion-c on-la-suprema-corte-de-la-provincia-de-buenos-aires-\%e2\%80\%8bor $\%$ e2\%80\%8b-la-emergencia-humanitari a-en-las-carceles-y-comisarias/

\section{Notas}

1 Esta es una práctica sumamente recurrente en las cárceles bonaerenses, considerada por muchos organismos de Derechos Humanos como una forma de tortura por la violencia que implica el traslado de una cárcel a otra, el cambio de compañeros/as de celda, el recibimiento en otras unidades y la suspensión de las actividades que desarrollaban en la unidad anterior.

2 Ciencias Jurídicas y Sociales, Periodismo y Comunicación Social y la Facultad de Humanidades y Ciencias de la Educación tienen programas específicos que trabajan con las y los estudiantes que se encuentran privados de su libertad en unidades cercanas a la Universidad Nacional de La Plata.

3 Todos los gráficos y datos sobre estudiantes privados/as de su libertad han sido aportados por el PAEPL.

4 Nos referimos al hecho de que el sistema penal y penitenciario reproduce violencias hacia las mujeres por su condición de mujeres, y también al hecho de que en la cárcel las desigualdades históricas entre varones y mujeres se recrudecen, y las condiciones de vida suelen ser más difíciles: tienen menos visitas, hay mayores niveles de violencia, entre otras.

5 Esto depende, por un lado, de la tradición de cada unidad en relación al centro de estudiantes como espacio de estudio y de encuentro entre estudiantes universitarios. Por otro lado, depende de la cantidad de alumnos/as en la escuela secundaria y el nivel de rotación entre penales, que atenta contra las trayectorias universitarias. 